\title{
QUANTUM INFORMATION, ENTANGLEMENT AND ENTROPY
}

Siddharth Sharma

$\underline{\text { Abstract }}$

In this review I had given an introduction to axiomatic Quantum Mechanics, Quantum Information and its measure entropy. Also, I had given an introduction to Entanglement and its measure as the minimum of relative quantum entropy of a density matrix of a Hilbert space with respect to all separable density matrices of the same Hilbert space. I also discussed geodesic in the space of density matrices, their orthogonality and Pythagorean theorem of density matrix.

\section{Postulates of Quantum Mechanics}

The basic postulate of quantum mechanics is about the Hilbert space formalism:

- Postulates 0: To each quantum mechanical system, has a complex Hilbert space $\mathbb{H}$ associated to it:

Set of all pure state, $\mathbb{H} / \sim:=\{\llbracket f \rrbracket \mid \llbracket f \rrbracket:=\{h: h \sim f\}\}$ and $h \sim f \Leftrightarrow \exists z \in \mathbb{C}$ such that $h=z f$ where $z$ is called phase

- Postulates 1: The physical states of a quantum mechanical system are described by statistical operators acting on the Hilbert space.

A density matrix or statistical operator, $\rho$ is a positive operator of trace 1 on the Hilbert space. Where $\rho$ is called positive if $\langle x, \rho x\rangle$ for all $x \in \mathbb{H}$ and $\langle *, *\rangle$.

- Postulates 2: The observables of a quantum mechanical system are described by selfadjoint operators acting on the Hilbert space.

A self-adjoint operator A on a Hilbert space $\mathbb{H}$ is a linear operator $A$ : $\mathbb{H} \rightarrow \mathbb{H}$ which satisfies

$$
\langle A x, y\rangle=\langle x, A y\rangle
$$

for $x, y \in \mathbb{H}$.

Lemma: The density matrices acting on a Hilbert space form a convex set whose extreme points are the pure states. 
Proof. Denote by $\boldsymbol{\Sigma}$ the set of density matrices. It is obvious that a convex combination of density matrices is positive and of trace one. Therefore, $\boldsymbol{\Sigma}$ is a convex set.

Lemma: Let

$$
\rho=\sum_{e \in \mathbb{B}}\left|x_{e}\right\rangle\left\langle x_{e}\left|=\sum_{h \in \mathbb{B}}\right| y_{h}\right\rangle\left\langle y_{h}\right|
$$

be decompositions of a density matrix and where $\mathbb{B}$ is the basis of Hilbert space, $\mathbb{H}$. Then there exists a unitary matrix $\left(U_{e h}\right)_{e h \in \mathbb{B}}$ such that

$$
\sum_{e \in \mathbb{B}} U_{e h}\left|x_{e}\right\rangle=\left|y_{h}\right\rangle
$$

Where,

$$
U_{e h}:=\frac{\left\langle x_{e} \mid y_{h}\right\rangle}{\sqrt{\left\langle x_{e} \mid x_{h}\right\rangle}}
$$

- Postulates 3: Let $\mathcal{X}$ be a finite set and for $x \in \mathcal{X}$ an operator $O_{x} \in \mathcal{B}(\mathbb{H})$ be given such that

$$
\sum_{x \in X} O_{x}^{*} O_{x}=i d_{\mathbb{H}}
$$

where $O_{x}^{*}$ is conjugate of $O_{x}$. Such an indexed family of operators is a model of a measurement with values in $\mathcal{X}$. If the measurement is performed in a state $\rho$, then the outcome $x \in \mathcal{X}$ appears with probability $\operatorname{tr}\left(O_{x}^{*} \rho O_{x}\right)$ and after the measurement the state of the system is

$$
\frac{O_{x}^{*} \rho O_{x}}{\operatorname{tr}\left(O_{x}^{*} \rho O_{x}\right)}
$$

If $\varphi: \mathcal{B}(\mathbb{H}) \rightarrow \mathbb{C}$ is a linear functional such that $\varphi(A) \geq 0$ if $A$ is positive and $\varphi(i d)=$ 1 , Then there exists a density matrix $\rho_{\varphi}$ such that,

$$
\varphi(A)=\operatorname{tr}\left(\rho_{\varphi} A\right)
$$

The functional $\varphi$ associates the expectation value to the observables $A$.

The density matrices $\rho$ and $\rho^{\prime}$ are called orthogonal if any eigenvector of $\rho$ is orthogonal to any eigenvector of $\rho^{\prime}$.

Let $\mathbb{B}$ be an orthonormal basis in a Hilbert space $\mathbb{H}$. The unit vector $\xi \in \mathbb{H}$ is complementary to the given basis if

$$
|\langle e, \xi\rangle|=\frac{1}{\sqrt{\operatorname{dim} \mathbb{H}}}
$$


For all $e \in \mathbb{B}$, where $\operatorname{dim} \mathbb{H}$ is dimension of $\mathbb{H}$.

Two orthonormal bases are called complementary if all vectors in the first basis are complementary to the other basis.

- Postulates 4: The composite system is described by the tensor product Hilbert space

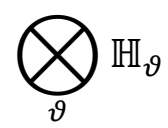

Given a density matrix $\rho$ on $\bigotimes_{\vartheta} \mathbb{H}_{\vartheta}$ there are density matrices $\rho_{\vartheta} \in \mathcal{B}\left(\mathbb{H}_{\vartheta}\right)$ such that

$$
\operatorname{tr}\left(\rho \bigotimes_{\gamma} \mathcal{A}_{\gamma}(\vartheta)\right)=\operatorname{tr}\left(\rho_{\vartheta} A_{\vartheta}\right)
$$

Where,

$$
\mathcal{A}_{\gamma}(\vartheta):=\left\{\begin{array}{l}
A_{\vartheta}, \gamma=\vartheta \\
i d_{\mathbb{H}_{\vartheta}}, \gamma \neq \vartheta
\end{array}\right.
$$

and $A_{\vartheta} \in \mathcal{B}\left(\mathbb{H}_{\vartheta}\right)$, here $\rho_{\vartheta}$ is called reduced density matrices.

Let,

$$
\rho=\bigotimes_{\vartheta} \rho_{\vartheta}
$$

Then we define $\operatorname{tr}_{\gamma}(\boldsymbol{\rho})$ as follows:

$$
\operatorname{tr}_{\gamma}(\rho):=\operatorname{tr}\left(\rho_{\gamma}\right) \bigotimes_{\vartheta \neq \gamma} \rho_{\vartheta}
$$

\section{$\underline{\text { Information and its Measures }}$}

- Shannon entropy: In his revolutionary paper Shannon proposed a statistical approach and he posed the problem in the following way: "Suppose we have a set of possible events whose probabilities of occurrence are $p_{1}, p_{2}, \ldots, p_{n}$. These probabilities are known but that is all we know concerning which event will occur. Can we find a measure of how much "choice" is involved in the selection of the event or how uncertain we are of the outcome?" Denoting such a measure by $H\left(p_{1}, p_{2}, \ldots, p_{n}\right)$, he listed three very reasonable requirements which should be satisfied, those three postulates are as follows:

a. Continuity: $H(p, 1-p)$ is a continuous function of $p$.

b. Symmetry: $H\left(p_{1}, p_{2}, \ldots, p_{n}\right)$, is a symmetric function of its variables. 
c. Recursion: For every $0 \leq \lambda<1$ the recursion $H\left(p_{1}, ., \lambda p_{r}, .,(1-\lambda) p_{r}, p_{n}\right)=$ $H\left(p_{1}, p_{2}, ., p_{r} . ., p_{n}\right)+p_{r} H(\lambda, 1-\lambda)$, holds

According to him there is only one function satisfying all this postulate is:

$$
H\left(p_{1}, p_{2}, ., p_{r} . ., p_{n}\right)=-\kappa \sum_{i=1}^{n} p_{i} \ln \left(p_{i}\right)
$$

Let $\left(X_{i}\right)_{i=1}^{n}$ be random variables with values in the set $\mathcal{X}:=\prod_{i=1}^{n} \mathcal{X}_{i}$. The following notation will be used.

a. $p\left(x_{i}\right)_{i=1}^{n}$ is probability of $\left(X_{i}\right)_{i=1}^{n}$ at $\left(x_{i}\right)_{i=1}^{n}$

b. $p\left(x_{i} \mid x_{j}\right)$ is probability of $X_{i}$ at $x_{i}$ knowing the probability of $X_{j}$. at $x_{j}$

Then,

$$
H\left(X_{i}\right)_{i=1}^{n}:=-\sum_{\left(x_{i}\right)_{i=1}^{n} \in \mathcal{X}} p\left(x_{i}\right)_{i=1}^{n} \ln p\left(x_{i}\right)_{i=1}^{n}
$$

Where if $n=2$

$$
H\left(X_{i}, X_{j}\right):=-\sum_{x_{i} \in X_{i}} \sum_{x_{j} \in X_{j}} p\left(x_{i}, x_{j}\right) \ln p\left(x_{i}, x_{j}\right)
$$

and

$$
p\left(x_{i}\right)_{i=1}^{n}=p\left(x_{2}\right) p\left(x_{1} \mid x_{2}, x_{3}, \ldots . x_{n}\right)
$$

Which leads to:

$$
p\left(x_{i}\right)_{i=1}^{n}=\frac{p\left(\left(\left(\left(x_{1} \mid x_{2}\right) \mid x_{3}\right) \mid \ldots\right) \mid x_{n}\right)}{p\left(x_{1}\right)} \prod_{i=1}^{n} p\left(x_{2}\right)
$$

Theorem: If $\left(X_{i}\right)_{i=1}^{n}$ are random variables of finite range, then

$$
H\left(X_{i}\right)_{i=1}^{n} \leq \sum_{i=1}^{n} H\left(X_{i}\right)
$$

Proof:

$$
H\left(X_{i}\right)_{i=1}^{n}:=-\sum_{\left(x_{i}\right)_{i=1}^{n} \in \mathcal{X}} p\left(x_{i}\right)_{i=1}^{n} \ln p\left(x_{i}\right)_{i=1}^{n}
$$

And 


$$
\sum_{i=1}^{n} H\left(X_{i}\right):=-\sum_{\left(x_{i}\right)_{i=1}^{n} \in \mathcal{X}} \prod_{i=1}^{n} p\left(x_{2}\right) \ln \prod_{i=1}^{n} p\left(x_{2}\right)
$$

And

$$
\prod_{i=1}^{n} p\left(x_{2}\right) \leq p\left(x_{i}\right)_{i=1}^{n}
$$

As

$$
p\left(x_{1}\right) \leq p\left(\left(\left(\left(x_{1} \mid x_{2}\right) \mid x_{3}\right) \mid \ldots\right) \mid x_{n}\right)
$$

Hence,

$$
H\left(X_{i}\right)_{i=1}^{n} \leq \sum_{i=1}^{n} H\left(X_{i}\right)
$$

Fano's inequality: Let $X$ and $Y$ be random variables such that their range is in a set of cardinality d and let $p:=\operatorname{Prob}(X \neq Y)$. Then

$$
H(X \mid Y) \leq p \log (d-1)+H(p, 1-p)
$$

- von Neumann Entropy: Let $\rho$ be density matrix of a quantum system then von Neumann Entropy of that quantum system:

$$
S(\rho):=-\kappa \operatorname{tr}(\rho \ln \rho)
$$

Theorem: Let $\rho$ and $\sigma$ be densities on a $d$-dimensional Hilbert space and let

$$
p:=\frac{\|\rho-\sigma\|_{1}}{2}
$$

where $\|*\|_{1}: \mathcal{B}(\mathbb{H}) \rightarrow \mathbb{R}_{0}^{+}$, is the norm on operator space $\mathcal{B}(\mathbb{H})$ of Hilbert space $\mathbb{H}$. Then,

$$
|S(\rho)-S(\sigma)| \leq p \log (d-1)+H(p, 1-p)
$$

Holds.

\section{- Quantum Relative Entropy:}

The relative entropy, or I-divergence of the probability distributions $p(x)$ and $q(x)$, is defined as

$$
D(p \| q):=\int_{-\infty}^{\infty} p(x) \ln \frac{p(x)}{q(x)} d x
$$


Assume that $\rho$ and $\sigma$ are density matrices on a Hilbert space $\mathbb{H}$, then

$$
S(\rho \| \sigma):=\left\{\begin{aligned}
\operatorname{tr} \rho(\ln \rho-\ln \sigma) & \text { if } \operatorname{supp}(\rho) \leq \operatorname{supp}(\sigma) \\
+\infty & \text { otherwise }
\end{aligned}\right.
$$

Hilbert-Schmidt inner product

$$
\Delta a=\rho a \sigma^{-1}
$$

For all $a \in \mathcal{B}(\mathbb{H})$

$$
\operatorname{tr} \rho(\ln \rho-\ln \sigma)=-\left\langle\rho^{\frac{1}{2}},(\ln \Delta) \rho^{\frac{1}{2}}\right\rangle
$$

where, $\langle A, B\rangle:=\operatorname{tr}\left(A^{*} B\right)$ and $A^{*}$ is conjugate of $A$

$$
\operatorname{tr} \rho(\ln \rho-\ln \sigma)=-\operatorname{tr}\left(\left(\rho^{\frac{1}{2}}\right)^{*}\left(\rho^{\frac{1}{2}}\right)(\ln \Delta)\right)
$$

Let $\rho \equiv e^{H}, \omega$ and $\sigma$ be three invertible densities. The $e-$ geodesic connecting $\rho$ and $\sigma$ is the curve

$$
\gamma_{e}(t):=\frac{e^{H+t A}}{\operatorname{tr}\left(e^{H+t A}\right)}
$$

for all $t \in[0,1]$, where $A:=\ln \sigma-\ln \rho$. Then, $\gamma_{e}(0)=\rho$ and $\gamma_{e}(1)=\sigma$. The $m-$ geodesic connecting $\rho$ and $\omega$ is the curve.

$$
\gamma_{m}(t):=\rho+t B
$$

for all $t \in[0,1]$ where $B:=\omega-\rho$. Then $\gamma_{m}(0)=\rho$ and $\gamma_{m}(1)=\omega$

Assume that the $e-$ geodesic connecting $\rho$ and $\sigma$ is orthogonal to the $m-$ geodesic connecting $\rho$ and $\omega$ with respect to the inner product

$$
\langle E, F\rangle_{\rho}:=\int_{0}^{\infty} \operatorname{tr}\left((s \mathbb{I}+\rho)^{-1} E^{*}(s \mathbb{I}+\rho)^{-1} F\right) d s
$$

A plain computation yields

$$
\begin{gathered}
S(\omega \| \rho)+S(\rho \| \sigma)-S(\omega \| \sigma)=\operatorname{tr}(A B)=\langle A, B\rangle_{H S} \\
T_{\rho}: X \mapsto \int_{0}^{\infty}(s \mathbb{I}+\rho)^{-1} X(s \mathbb{I}+\rho)^{-1} d s
\end{gathered}
$$

Them According Dénes Petz

$$
\langle X, Y\rangle_{H S}=\left\langle T_{\rho}(X), Y\right\rangle_{\rho}
$$

And,

$$
T_{\rho}(A)=\dot{\gamma}_{e}(0)
$$


It is obvious that:

$$
B=\dot{\gamma}_{m}(0)
$$

Therefore

$$
\langle A, B\rangle_{H S}=\left\langle T_{\rho}(A), B\right\rangle_{\rho}=\left\langle\dot{\gamma}_{e}(0), \dot{\gamma}_{m}(0)\right\rangle_{\rho}
$$

and we can conclude that if our assumption holds then that implies:

$$
\left\langle\dot{\gamma}_{e}(0), \dot{\gamma}_{m}(0)\right\rangle_{\rho}=0 \Rightarrow S(\omega \| \rho)+S(\rho \| \sigma)=S(\omega \| \sigma)
$$

This is some time called Pythagorean theorem of density matrix.

\section{- Renyi Entropy, Quantum Renyi Entropy and Quantum Relative Renyi Entropy:}

The Renyi entropy of order $\alpha \neq 1$ of the probability distribution $\left(p_{1}, p_{2}, \ldots, p_{n}\right)$ is defined by

$$
H_{\alpha}\left(p_{1}, p_{2}, \ldots, p_{n}\right)=\frac{1}{1-\alpha} \ln \sum_{k=1}^{n} p_{k}^{\alpha}
$$

The Quantum Renyi entropy of order $\alpha \neq 1$ of the density matrix $\rho$ is defined by

$$
S_{\alpha}(\rho)=\frac{1}{1-\alpha} \ln \operatorname{tr}\left(\rho^{\alpha}\right)
$$

The Quantum Renyi entropy of order $\alpha \neq 1$ of the density matrix $\rho$ with respect $\sigma$ to is defined by

$$
S_{\alpha}(\rho \| \sigma)=\frac{1}{\alpha-1} \ln \operatorname{tr}\left(\rho^{\alpha} \sigma^{1-\alpha}\right)
$$

\section{Entanglement}

Let $\mathcal{B}\left(\mathbb{H}_{A}\right)$ and $\mathcal{B}\left(\mathbb{H}_{B}\right)$ be the algebras of bounded operators acting on the Hilbert spaces $\mathbb{H}_{A}$ and $\mathbb{H}_{B}$. The Hilbert space of the composite system is $\mathbb{H}_{A B}:=\mathbb{H}_{A} \otimes \mathbb{H}_{B}$. The algebra of the operators acting on $\mathbb{H}_{A B}$ is $\mathcal{B}\left(\mathbb{H}_{A B}\right):=\mathcal{B}\left(\mathbb{H}_{A}\right) \otimes \mathcal{B}\left(\mathbb{H}_{B}\right)$.

In the vector space $\mathcal{B}(\mathbb{H})$ the standard positive cone is the set of all positive matrices. This cone induces the partial ordering.

$$
A \leq B \Leftrightarrow\langle\eta, A \eta\rangle \leq\langle\eta, B \eta\rangle \text { for every vector } \eta
$$

In the product space $\mathcal{B}\left(\mathbb{H}_{A B}\right):=\mathcal{B}\left(\mathbb{H}_{A}\right) \otimes \mathcal{B}\left(\mathbb{H}_{B}\right)$, we have two natural positive cones, $\mathcal{B}\left(\mathbb{H}_{A B}\right)^{+}$consists of the positive matrices acting on $\mathbb{H}_{A B}:=\mathbb{H}_{A} \otimes \mathbb{H}_{B}$, and the cone $\mathcal{S}$ consists of all operators of the form 


$$
\sum_{i} A_{i} \otimes B_{i}
$$

Where $A_{i} \in \mathcal{B}\left(\mathbb{H}_{A}\right)^{+}$and $B_{i} \in \mathcal{B}\left(\mathbb{H}_{B}\right)^{+}$. It is obvious that $\mathcal{S} \subset \mathcal{B}\left(\mathbb{H}_{A B}\right)^{+}$. A state is called separable (or unentangled) if its density belongs to $\mathcal{S}$. And if not then they are called entangled states.

pure states are extreme points in the state space. If a pure state is a convex combination $\sum_{i} p_{i} P_{i} \otimes B_{i}$ of product pure states, then this convex combination must be trivial, that is, $P \otimes Q$.

Lemma: Any unit vector $\Psi \in \mathbb{H}_{A B}$ can be written in the form

$$
\Psi \in \sum_{k} \sqrt{p_{k}} g_{k} \otimes h_{k}
$$

where the vectors $g_{k} \in \mathbb{H}_{A}$ and $h_{k} \in \mathbb{H}_{B}$ are pairwise orthogonal and normalized; moreover $\left(p_{k}\right)$ is a probability distribution. This expansion of $\Psi$ is called Schmidt decomposition.

Let $\operatorname{dim} \mathbb{H}_{A}=\operatorname{dim} \mathbb{H}_{B}=n$. A pure state $|\Phi\rangle\langle\Phi|$ on the Hilbert space $\mathbb{H}_{A} \otimes \mathbb{H}_{B}$ is called maximally entangled if the following equivalent conditions hold:

a. The reduced densities are maximally mixed states.

b. When the vector $|\Phi\rangle$ is written in the form, then $p_{k}=n^{-1}$ for every $1 \leq k \leq n$.

c. There is a product basis such that $|\Phi\rangle$ is complementary to it.

The density matrix of a maximally entangled state on $\mathbb{C}^{n} \otimes \mathbb{C}^{n}$ is of the form

$$
\rho=\frac{1}{\sqrt{n}} \sum_{\mu \nu} e_{\mu \nu} \otimes e_{\mu \nu}
$$

\section{- Entanglement Measures:}

The degree of entanglement of a state $\rho_{\mathrm{AB}}$ on the bipartite Hilbert space $\mathbb{H}_{A B}:=\mathbb{H}_{A} \otimes$ $\mathbb{H}_{B}$ is its distance from the convex set of separable states. One possibility is to use the relative entropy as a distance function. In this way, we can arrive at the concept of the relative entropy of entanglement:

$$
E_{R E}\left(\rho_{\mathrm{AB}}\right):=\inf \left\{S\left(\rho_{\mathrm{AB}}|| D\right): D \in \mathcal{S}\right\}
$$

where $D$ runs over the set of separable states on $\mathbb{H}_{A B}:=\mathbb{H}_{A} \otimes \mathbb{H}_{B}$. If $\rho_{\mathrm{AB}}$ is faithful, then the minimizer is unique and can be called "the best separable approximation of $\rho_{\mathrm{AB}}$. 


\section{$\underline{\text { References }}$}

- S. AMARI AND H. NAGAOKA, Methods of information geometry, Transl. Math. Monographs 191, AMS.

- H. ARAKI, Relative entropy for states of von Neumann algebras, Publ. RIMS Kyoto Univ. 11(1976), 809-833

- J. ACZEL AND' Z. DAROCZY 'On measures of information and their characterizations, Academic Press, New York, San Francisco, London

- O. BRATTELI AND D. W. ROBINSON, Operator Algebras and Quantum Statistical Mechanics II, Springer-Verlag, New York-Heidelberg-Berlin

- M. B. PLENIO, S. VIRMANI AND P. PAPADOPOULOS, Operator monotones, the reduction criterion and relative entropy, J. Physics A 33(2000), L193-197.

- DÉNES PETZ, Quantum Information Theory and Quantum Statistics, Theoretical and Mathematical Physics Series, Springer

- A. RENYI ' On measures of entropy and information, in Proceedings of the 4th Berkeley conference on mathematical statistics and probability, ed. J. Neyman, pp. 5 J. $\mathrm{R}^{`}$ EHA' CEK AND ` Z. HRADIL, Quantification of entanglement by means of convergent iterations, Phys. Rev. Lett. 90(2003), 127904.47-561, University of California Press, Berkeley 